\title{
Missing Value Imputation using Hybrid Higher Order Neural Classifier
}

\author{
Lipismita Panigrahi ${ }^{1}$, Kaberi Das ${ }^{2}$ and Debahuti Mishra ${ }^{3^{*}}$ \\ 'Computer Science \& Information Technology, Balasore College of Engineering and Technology, Balasore, Odisha, India \\ ${ }^{2}$ Department of Computer Applications, Institute of Technical Education and Research, Siksha O Anusandhan \\ Deemed to be University, Bhubaneswar, Odisha, India \\ ${ }^{3}$ Computer Science \& Engineering, Institute of Technical Education and Research, Siksha O Anusandhan \\ Deemed to be University, Bhubaneswar, Odisha, India; debahutimishra@soauniversity.ac.in
}

\begin{abstract}
Missing values can cause serious problems while mining data sets, such as i) loss of information and efficiency; ii) problem in data handling computation and analysis due to irregularities in the data patterns and non-applicability of standard software; and iii) serious bias if there are systematic differences between the observed and the unobserved data. Missing values can also cause misleading results by introducing bias. This paper focuses on a methodological framework for the development of an automated data imputation model based on Hybrid Higher Order Neural Network Classifier (HHONC). Four real, integer and simulated data sets are exposed to a perturbation experiment, based on the random generation of missing values. Here different imputation methods are applied in glass identification, wine recognition, heart disease and lung cancer data set to find the missing value and compared with different classic imputation procedures. The experiment not only improves the quality of a database with missing value but also the best results are clearly obtained with different variables.
\end{abstract}

Keywords: Hybrid Higher Order Neural Classifier (HHONC), Imputation Method, Missing Value, Neural Network(NN)

\section{Introduction}

Missing data or missing values occur when no data value is stored for the variable in the current observation. Inconsistent values are produced when the answer is not accurately recorded and for these data sets usually we find errors. To deal with missing data, many analytic tools are used to fill the missing values with a value estimated by some process, this process is called imputation. To suggest values for missing data before training a classifier some imputation techniques are used. Some single imputation is mean/mode ${ }^{1}$ which replaces the missing value with the mean value of the attribute over all instances; regression ${ }^{2}$ and hot-deck imputation. So the experimental evaluation shows that these simple imputation methods typically do not significantly improve the machine learners performance. We have therefore used Hybrid Higher Order Neural Networks (HHONC), which uses better imputation techniques for filling in the missing values before training the classifiers. Neural Network (NN) have several limitations, neither it excel in discontinues or non-smooth ${ }^{3}$, nor it deal well on incomplete or noisy data ${ }^{4}$. Higher Order Neural Networks (HONNs) can be considered to improve the various limitations of traditional neural network. HONNs make a higher order correlation among inputs ${ }^{5}$ by combining inputs nonlinear. HONN successfully provides an efficient open-box model of nonlinear input-output mapping which provides easier understanding of data mining. HONNC structures are simpler than NN and initialization of learning parameters (weights) will not be catastrophic. Furthermore, HONNs use different activation functions such as (sigmoid, sine, cosine, SINC, etc.) for better fitting specifications. Moreover, HONNs most often run faster

${ }^{*}$ Author for correspondence 
than NNs. Examples in implementation of two-input and three-input XOR functions by using second-order neural network (SONN) proved that SONN is several times faster than $\mathrm{NN}^{6}$. The layout of this paper is as follows; section 2 deals with related work, in section 3 the preliminary concepts of data imputation, HHONC has been described, in section 4 schematic representations of proposed model is given, in section 5 experimental evaluations and results are described and finally, section 6 deals with conclusion and future work.

\section{Related Work}

Bishop $^{7}$ introduced data imputation as problem of estimation of missing values using artificial neural networks. Rumelhart ${ }^{8}$ designed back-propagation network (BPN). Moody ${ }^{9}$ designed radial basis function (RBF). Kohonen ${ }^{10}$ introduced self-organization map (SOM). Lee $^{11}$ proposed a fast self-organizing hierarchical cerebella model arithmetic computer (HCMAC). Little ${ }^{12}$ introduced several desirable features for multiple imputations. Shin ${ }^{13}$ have introduced an efficient higher order neural network as a PSN model. Fallahnezhad ${ }^{14}$ introduced a hybrid higher order neural classifier for handling classification problems.

\section{Preliminaries}

\subsection{Data Imputation Methods}

Let us consider a data set $T$, disposed as data matrix where $p$ variables have been measured in each one of $n$ records, defining a matrix $n \times p$, but the data collecting process may have produced missing values for some cells of $T$. This $T$ is an approximation to the true data set $D$, where the $n \times p$ values would have been completely recorded. A data imputation model is defined by a set of rules and procedures to obtain an approximation $D *$ to $D$ working on the available data set $T$. The existing methods for imputation are mean $/$ mode $^{15}$, regression method ${ }^{16,17}$ and hot-deck ${ }^{15}$. Missing data can appear by different mechanisms or with different patterns. There are three types of missing data mechanisms i.e. MAR (missing at random), MCAR (missing completely at random), NMCAR (Not missing completely at random $)^{12,16}$.

\subsection{Hybrid Higher Order Neural Classifier (HHONC)}

In data imputation problem each categorical variable must be codified by a vector formed by dummy variables $0-1$, one for each class, so the number of inputs $p$ is usually larger than the number of variables in the data file. The number of outputs of the $\mathrm{NN}$ is equal to the number of inputs, and thus the size of the network is $(p, H, p)$, where $H$ is hidden layer. High-order concept is widely used in neuron type (linear, power, multiplicative, sigmoid, etc.), neuron activation function type (polynomial, sigmoid, cosine, sine, SINC, etc. $)^{14,6}$. HONNC uses a higher correlation of input neurons for better fitting properties which often leads to a higher number of learning parameters (weights). The general sigma- $p i^{13}$ model has three type of structures $1 \mathrm{~b}, 0$ and 1 model including two features, $x_{1}$ and $x_{2}$ as given in (1), (2) and (3).

HONN Model 1b:

$$
Z=\sum_{i, j=0}^{N} a_{i j}^{0}\left\{a_{i j}^{k x_{1}} \cdot f_{i}^{x_{1}} a_{i}^{x_{1}}\left(x_{1}\right)\right\} \cdot\left\{a_{i j}^{k x_{2}} \cdot f_{j}^{x_{2}} a_{j}^{x_{2}}\left(x_{2}\right)\right\}
$$

\section{HONN Model 1:}

$$
Z=\sum_{i, j=0}^{N} a_{i j}^{0}\left\{f_{i}^{x_{1}} a_{i}^{x_{1}}\left(x_{1}\right)\right\} \cdot\left\{f_{j}^{x_{2}} a_{j}^{x 2}\left(x_{2}\right)\right\}
$$

HONN Model 0:

$$
Z=\sum_{i, j=0}^{N} a_{i j}^{0}\left\{f_{i}^{x_{1}}\left(x_{1}\right)\right\} \cdot\left\{f_{j}^{x_{2}}\left(x_{2}\right)\right\}
$$

Learning algorithm of HHONC: THONN (Trigonometric Higher Order Neural Network) model: The activation functions are trigonometric (sine \& cosine) functions $f\left(x_{1}\right)=\cos \left(x_{1}\right)$ and $f\left(x_{2}\right)=\sin \left(x_{2}\right)$. SINCHONN (SINC Higher Order Neural Network): Here the activation functions are SINC functions; $f(x)=\frac{\sin (x)}{x}$. SPHONN (Sigmoid Polynomial Higher Order Neural Network): Here the activation functions are SIGMOID functions. Both LOGSIG and TANSIG functions ${ }^{14}$ are implemented by HONN model 0 in first layer. In second layer, linear combinations of these units are chosen. 


\section{$3.3 k$-nearest-neighbor Classifier $(k-\mathrm{NN})$}

It finds the closest $k$-neighbours from the unknown instance and returns the average value of the real-valued labels of the neighbours. By Euclidean distance the closeness of the neighbours is defined for continuous attributes and Hamming distance for discrete ones. The distance of the attribute is zero when the two instances miss the values of the same attribute, but when only one has a missing value, a maximal distance is assigned.

\section{Schematic Representation of Proposed Model}

Proposed model make a comparison study between HHONC and different imputation techniques, for imputation of randomly generate missing data. Here $70 \%$ of data $^{18}$ are used for training and $30 \%$ of data for testing. The total procedure is illustrated in Figure 1.

\section{Experimental Evaluation and Result}

Step 1: Data Sets: Our experimental evaluation has been conducted on four data sets. A brief description of each data $\operatorname{set}^{18}$ is shown in Table 1 . Where, name: name of the data set; size: number of records; Inputs/outputs: total number of inputs and outputs of the HHONC model; NQV: number of quantitative variables; NCV: number of categorical variable.

Step 2: Pre-processing the data sets: Several pre-processing tasks have been performed for each data set. Firstly, all the constant variables across the data file are deleted. Secondly the quantitative variables are normalized, using Z-score normalization ${ }^{15}$.

Step 3: Error generation: Then we are moving towards error generation. Let we assume that the data set is complete and correct, so introducing random errors a perturbed data file is obtained. Thus, a variable $Y_{\mathrm{d}}$ has been defined which is a disturbed variable for each original variable $Y$.

Step 4: HHONC: Then the training data has been applied to HHONC. The order of each high-order unit dynamically changes in training process (decrease and increase). At the output stage there is a shifted hard-limit function ${ }^{14}$. This unit is just used in testing process and it's because of our binary bit coding $(0,1)$ for identifying different classes. The output of HHONC [14] during the training process is shown by $Z$ and the output of HHONC during test samples process is shown by $Z$. Hence, (4) and (5) mathematically represents $\mathrm{HHONC}$ outputs as a function of input features.

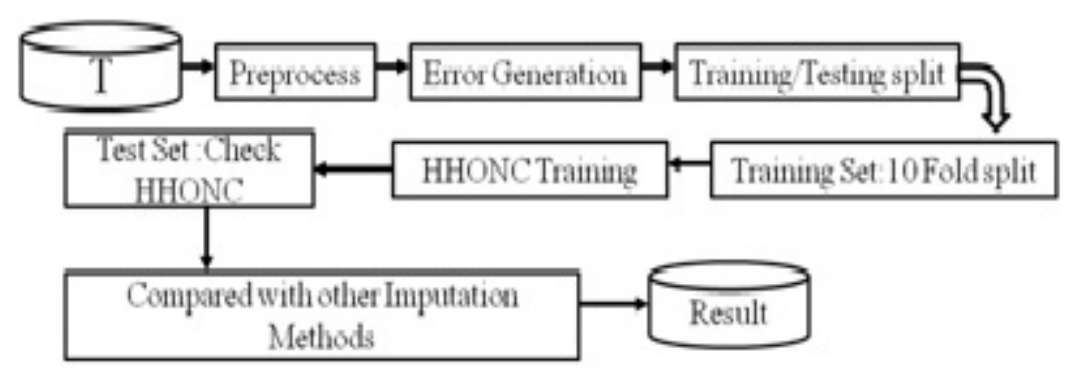

Figure 1. Schematic representation of proposed work.

Table 1. Data sets used in the data imputation experiments

\begin{tabular}{lcccc}
\hline Data Sets & Size & Input/output & NQV & NCV \\
\hline Glass & 214 & 9 & 9 & 0 \\
Wine & 178 & 13 & 13 & 0 \\
Lung & 32 & 56 & 56 & 0 \\
Heart & 303 & 75 & 6 & 7 \\
\hline
\end{tabular}




$$
\begin{gathered}
N_{k} \\
z_{i}=Y \times V=v_{0}+\sum_{j=1}^{k} v_{j i} y_{j}(\bar{x}), \quad Z=\left[z_{1}, \ldots, z_{i}, \ldots, z_{L}\right]
\end{gathered}
$$

Where, $L$ is the number of outputs (bit number of output binary code), $P$ is the number of features, $K$ is the number of higher order units. The number of hidden neurons in first layer is directly specified by the order of high-order units. Second hidden layer of HHONC is a linear order and experiments reveal that the desired accuracy can be reached if the number of hidden neurons of this layer is chosen to be same as the number of bits that output is coded. The first and second layer weights are updated by (7) and (9), respectively.

$$
\begin{gathered}
W_{j i_{1}-i_{K_{k}}}^{(k)}(t+1)=W_{j i_{1}-i_{N_{k}}}^{(k)}(t)-\eta \cdot\left(\partial E / \partial W_{j i_{1}-i_{N_{k}}}^{(k)}\right) \\
\frac{\partial E}{\partial y_{j}}=\frac{\partial}{\partial y_{j}}\left[\frac{1}{2} \sum_{i=1}\left(d_{i}-z_{l}\right)^{2}\right] \\
=\sum_{i=1}\left(d_{i}-z_{l}\right)^{2} \frac{\partial}{\partial y_{j}}(Y \times V)=\sum_{i=1}^{l}\left(d_{l}-z_{l}\right) \mathrm{v}_{j l} \\
v_{j i}(t+1)=\mathrm{v}_{j l}(t)-\eta \cdot\left(\partial E / \partial V_{j l}\right)
\end{gathered}
$$

$$
\frac{\partial E}{\partial v_{j l}}=\frac{\partial E}{\partial z_{l}} \cdot \frac{\partial z_{l}}{\partial v_{j l}}=\frac{\partial E}{\partial z_{l}} \cdot y_{j}=y_{j} \cdot \sum_{i=1}^{L}\left(d_{l}-z_{l}\right)
$$

Where, $t$ is training state, $\eta$ is learning coefficient, $E$ is error, $W$ is weight matrix of first hidden layer and $V$ is weight matrix of second hidden layer, $L$ is the number of outputs (bit number of output binary code), $P$ is the number of input samples, $K$ is the number of higher order units. The result shows the comparison of the different methods for missing data imputation, classic imputation procedures such as mean/mode, regression, $k$-NN, ANN and HHONC-based model on the different data sets in Table 2, it can be seen that the proposed HHONC model shows better percentage of accuracy in comparison to other method.

Table 2. Accuracy of different models

\begin{tabular}{lccccc}
\hline Datasets & Mean/Mode & Regression & $\boldsymbol{k}$-NN & ANN & HHONC \\
\hline Heart & 73.9 & 72.4 & 80.0 & 90.1 & 99.5 \\
Lung & 98.9 & 99.0 & 98.6 & 98.4 & 99.1 \\
Wine & 98.5 & 99.0 & 98.7 & 98.5 & 99.7 \\
Glass & 99.3 & 99.4 & 99.2 & 99.9 & 99.0 \\
\hline
\end{tabular}

\subsection{Output of the Dataset after Normalization}

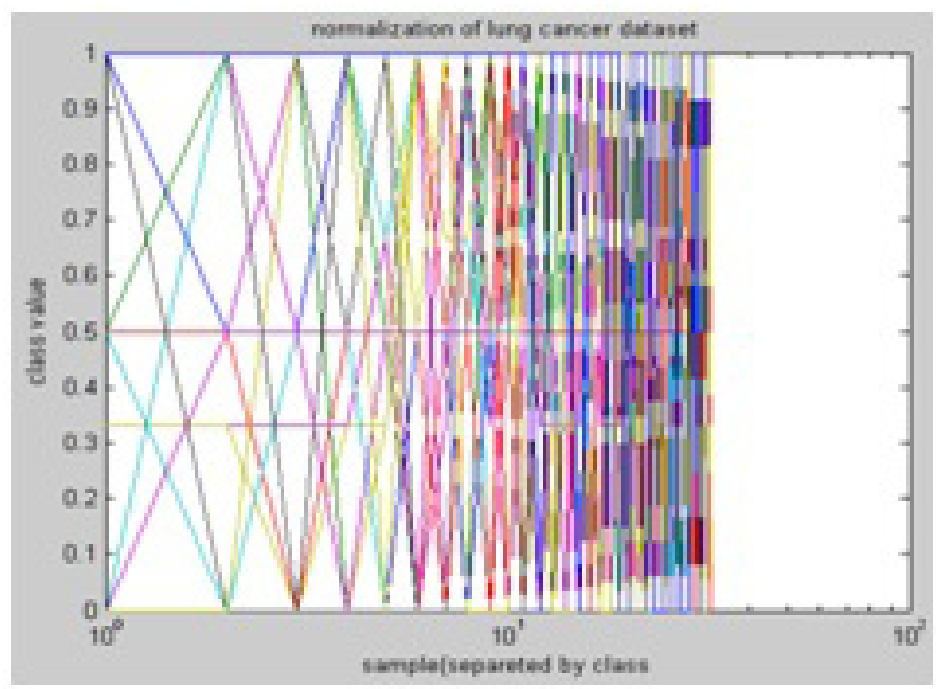

Figure 2(a). Normalization of lung cancer data set. 


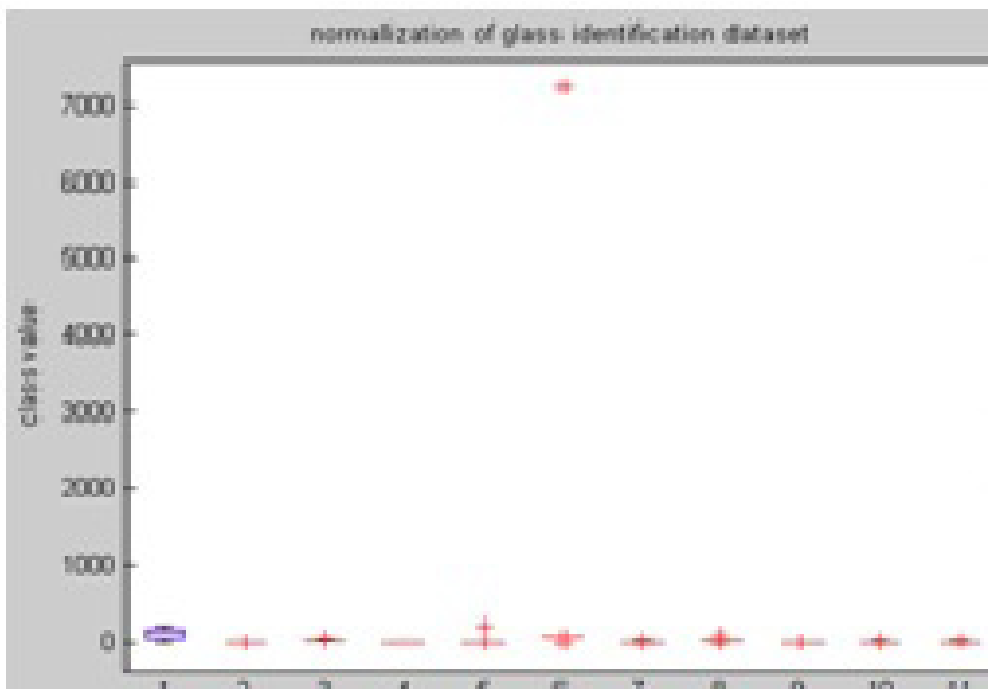

Figure 2(b). Normalization of glass identification data set.

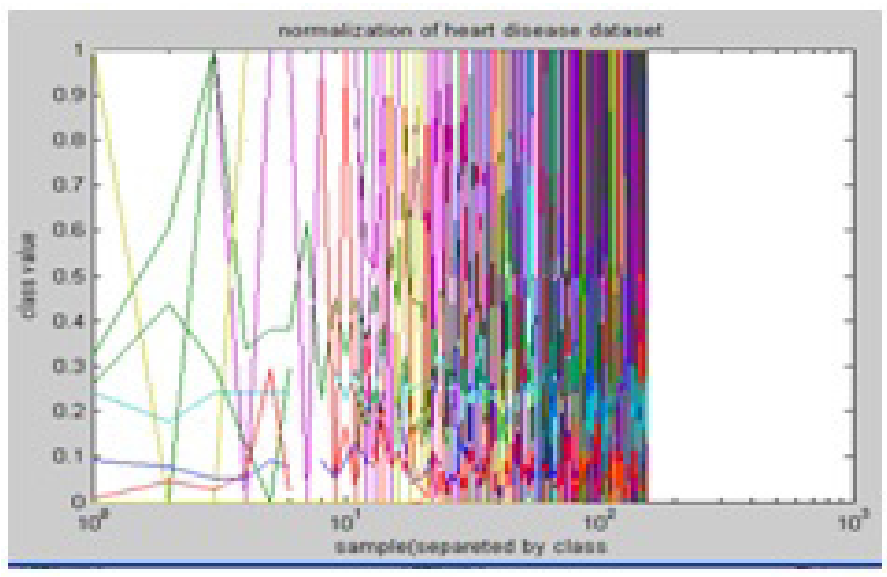

Figure 2(c). Normalization of heart disease data set.

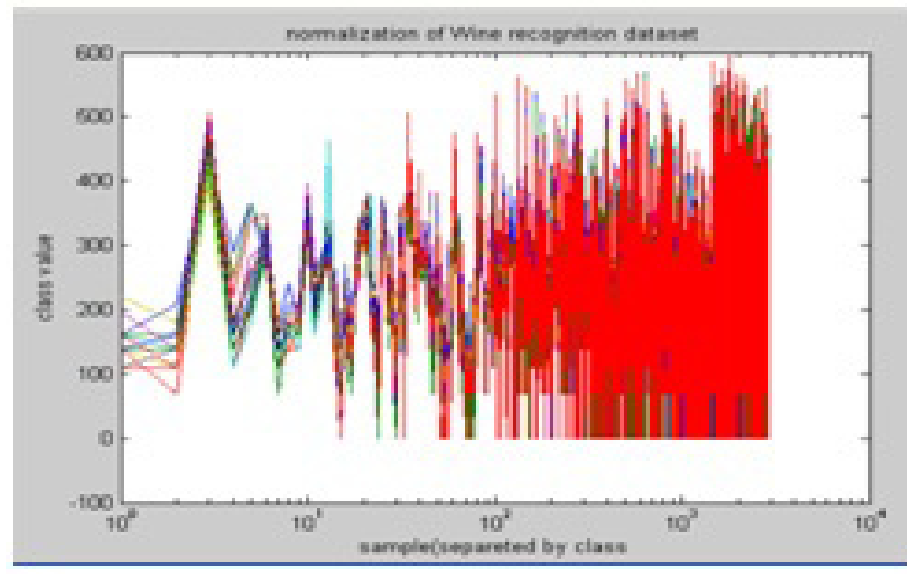

Figure 2(d). Normalization of wine recognition data set. 


\subsection{Output of Complete Dataset after Imputation of Missing Values}

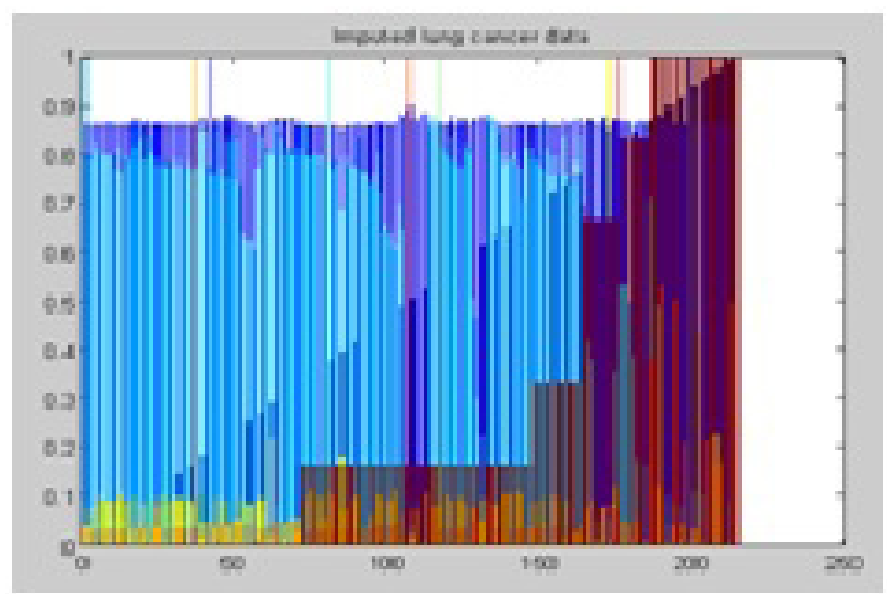

Figure 3(a). Lung cancer data set by different imputation methods.

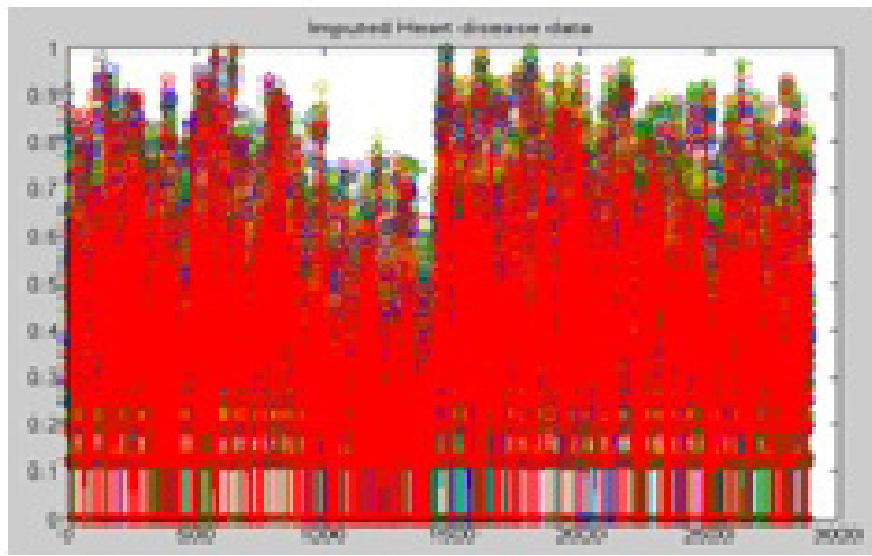

Figure 3(b). Heart disease data set by different imputation methods.

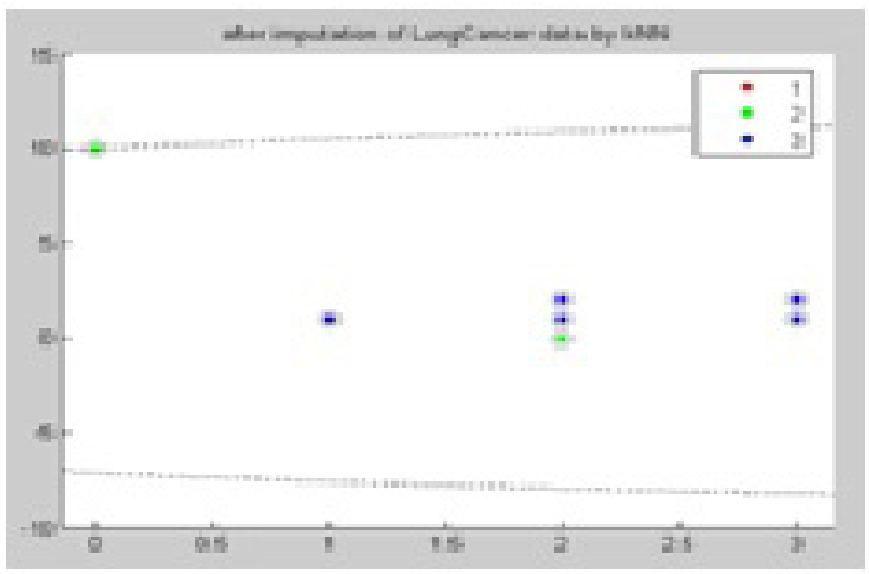

Figure 3(c). Result of imputation on missing data using $k$-NN.

Here in the graph 1-3 shows the class label of lung cancer data set. 


\subsection{Comparison of Different Imputation Models with HHONC}

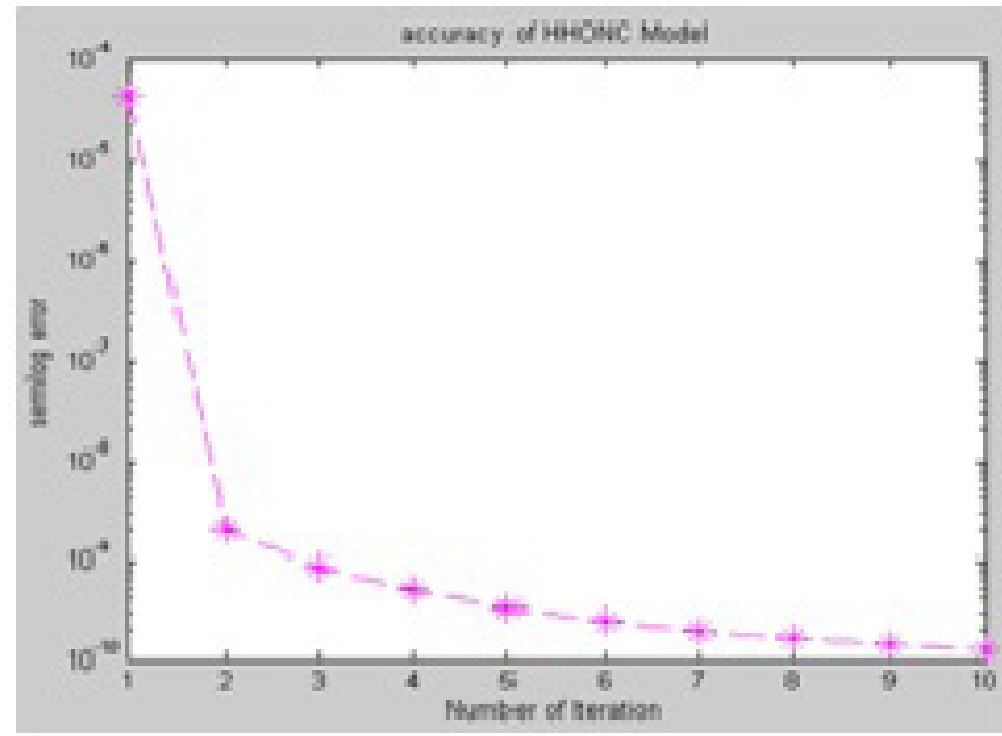

Figure 4. Accuracy of HHONC model

\section{Conclusion and Future Work}

In this paper, proposed HHONC model has been used for imputing the missing value and compare with four classic methods: mean/mode imputation, regression models, $k$-NN and ANN. The proposed method used here shows the high average classification accuracy rates in comparison to the other existing methods. From the experimental result we conclude that HHONC produce more accuracy while working with the four real and simulated data sets. By using hybrid components of different HONN units and decreasing the number of learning parameters (weights) instead of fully-connected HONN, we find a HHONC model accurately fitted over each dataset. For more fussiness in future we can include adaptive higher order functions or different wavelet functions as the kernel of HHONC instead of each neuron activation function and can apply feature reduction on it.

\section{References}

1. Witten IHF. Practical machine learning tools and techniques. San Francisco: Morgan Kaufmann; 2005.

2. Cessie SLe, Houwelingen JCV. Ridge Estimators in Logistic Regression. Applied Statistics. 1992; 41:191-201.
3. Fulcher J, Zhang M, Xu S. Application of higher-order neural networks to financial time series prediction. Artificial neural networks in finance and manufacturing. New York: Idea Group Inc.(IGI); 2006. p. 80-108.

4. Dong G, Pei J. Sequence data mining. Springer; 2007.

5. Zurada JM. Introduction to artificial neural systems. St. Paul, West Publishing Company; 1992.

6. Gupta MM, Homma N, Hou Z, Solo AMG, Goto. Fundamental theory of artificial higher order neural networks. Artificial higher order neural networks for economics and business. 2009; 368-88.

7. Bishop C, Ripley B. Neural networks for pattern recognition. Oxford University, Cambridge University; 1995, 1996.

8. Rumelhart DE, Hinton GE, Williams RJ. Learning representation by back-propagation errors. 2007; 323:533-6

9. Moody J, Darken C. Fast learning in networks of locally tuned processing units. 1989; 281-94.

10. Kohonen T. Self-organization and associative memory. Springer-Verlag; 1998. p. 119-57.

11. Lee HM, Chen CM, Lu YF. A self-organizing HCMAC neural network classifier. IEEE Transaction on Neural Networks. 2003; 14:15-27.

12. Little R, Rubin D. Statistical analysis with missing data. New York: John Wiley and Sons; 1987.

13. Shin Y, Ghosh J. The pi-sigma networks: An efficient higher-order neural network for pattern classification and function approximation; Proceedings of International Joint Conference on Neural Networks; 1991. p. 13-8. 
14. Fallahnezhad M, Moradi MH, Zaferanlouei S. A Hybrid Higher Order Neural Classifier for handling classification problems. Expert Systems with Applications. 2011; 38:386-93.

15. Ramírez EL, Mejías RP, Coello ML, Vega MD. Missing value imputation on missing completely at random data using multilayer perceptrons. Neural Networks. 2011; 24:121-9.
16. Howell DC, TW, Turner S. The analysis of missing data, Handbook of Social Science Methodology. Outhwaite, London: 2007.

17. Scott AJ. Illusions in regression analysis. International Journal of Forecasting. 2012.

18. UCI machine learning repository. Available from: http:// www.ics.uci.edu/ mlearn/MLRepository.html 2012. 